\title{
Efficacy of lapatinib combined with capecitabine in patients with HER2-positive metastatic breast cancer in a real-world study
}

\author{
XINYU GUI, HUIPING LI, YING YAN and RUYAN ZHANG \\ Key Laboratory of Carcinogenesis and Translational Research (Ministry of Education/Beijing), Department \\ of Breast Oncology, Peking University Cancer Hospital and Institute, Beijing 100142, P.R. China
}

Received May 16, 2020; Accepted October 8, 2020

DOI: $10.3892 / \mathrm{ol} .2020 .12241$

\begin{abstract}
The aim of the present study was to determine the efficacy and safety of lapatinib-based treatment for patients with human epidermal growth factor receptor-2-positive $\left(\mathrm{HER}^{+}\right)$metastatic breast cancer $(\mathrm{MBC})$. The aim of the present real-world study was to investigate the medical records and follow-up information of 92 patients with $\mathrm{HER}^{+}{ }^{+} \mathrm{MBC}$ who received a lapatinib-based regimen at the recurrent/metastatic stage, 78 of whom had been pretreated with trastuzumab. The results demonstrated that the median progression-free survival (PFS) was 5.8 months and the overall survival (OS) was 21.5 months, with an objective response rate (ORR) of $21.7 \%$, disease control rate (DCR) of $87.0 \%$ and clinical benefit rate (CBR) of $47.8 \%$. In the patients receiving a lapatinib-based regimen as first-, second- and third/later-line treatment, the median PFS was 10.4, 5.2 and 5.1 months $(\mathrm{P}=0.048)$, the median $\mathrm{OS}$ was 32.9, 29.1 and 13.0 months $(\mathrm{P}<0.001)$, the ORR was 38.9, 23.3 and $13.60 \%$, and the DCR was $100,83.3$ and $84.1 \%$, respectively. In the trastuzumab-resistant $(n=71)$ and trastuzumab-sensitive $(n=21)$ patients, the median PFS was 5.2 and 9.1 months $(\mathrm{P}=0.032)$, and the median OS was 21.4 and 44.3 months $(\mathrm{P}=0.003)$, respectively. In the patients who received lapatinib plus chemotherapy $(n=68)$, the median PFS with lapatinib plus capecitabine $(n=38)$ was 8.1 months, as compared with the 5.1 months with lapatinib plus other chemotherapy agents $(n=30 ; P=0.005)$. The median PFS of 14 patients with brain metastases was 8.4 months, with an ORR of $35.7 \%$ and a DCR of $85.7 \%$. Multivariate analysis revealed that the line of lapatinib-based treatment and its combination with capecitabine or
\end{abstract}

Correspondence to: Professor Huiping Li, Key Laboratory of Carcinogenesis and Translational Research (Ministry of Education/Beijing), Department of Breast Oncology, Peking University Cancer Hospital and Institute, 52 Fucheng Road, Haidian, Beijing 100142, P.R. China

E-mail: huipingli2012@hotmail.com

Key words: human epidermal growth factor receptor-2-positive, metastatic breast cancer, lapatinib, capecitabine, progression-free survival a different agent were independent prognostic factors for the median PFS in patients with HER2 ${ }^{+}$MBC. A limited number of adverse events were observed with the combination of lapatinib and capecitabine. Therefore, the findings of the present study suggested that lapatinib-based treatment is effective in patients with HER2 ${ }^{+}$MBC (even in trastuzumab-pretreated patients), and the combination of lapatinib with capecitabine may be recommended due to its good efficacy, convenience and tolerability.

\section{Introduction}

Human epidermal growth factor receptor-2 (HER2) is overexpressed in $20-25 \%$ of patients with breast cancer, and anti-HER2 targeted therapy is extensively used in patients with HER $2^{+}$breast cancer. However, there is a need for alternative treatments, as the majority of patients with $\mathrm{HER}^{+}$metastatic breast cancer (MBC) eventually develop resistance to trastuzumab (1). As a prognostic and predictive factor, HER2 amplification/overexpression has been proven to contribute to the development of central nervous system (CNS) metastases (2-4). Chemotherapy is not routinely administered to patients with CNS metastases from breast cancer, as the majority of anticancer agents do not cross the blood-brain barrier. Patients with breast cancer who develop CNS disease have a poor prognosis, with a 1-year survival rate of $20 \%$, which makes CNS metastasis a significant consideration for oncologists with regards to treatment (5).

As an oral small molecule and dual tyrosine kinase inhibitor of epidermal growth factor receptor and HER2, lapatinib interferes with signal transduction pathways involved in tumour cell proliferation and growth to exert an antitumor effect on HER2 ${ }^{+}$breast cancer $(6,7)$. Lapatinib in combination with capecitabine was approved on March 13, 2007 by the U.S. Food and Drug Administration for the treatment of patients with HER2 ${ }^{+} \mathrm{MBC}$ who had received prior treatment that included an anthracycline, a taxane and trastuzumab (8). In addition, certain studies have reported the efficacy and safety of lapatinib plus chemotherapy or endocrine therapy as first-line treatment in patients with previously untreated brain metastases from HER $2^{+} \mathrm{MBC}$ and newly diagnosed HER2 ${ }^{+}$MBC (9-11). Brain metastases develop in $30-50 \%$ of patients with HER2 ${ }^{+}$MBC. The combination of lapatinib and capecitabine has confirmed CNS antitumor activity in 
HER $2^{+}$breast cancer, which may promote the design of novel targeted approaches to treatment in selected patients $(9,12,13)$. The present real-world clinical study was designed to evaluate the efficacy and tolerability of a lapatinib-based regimen in patients with HER2 ${ }^{+} \mathrm{MBC}$.

\section{Patients and methods}

Study population. The medical records of adult patients with HER $2^{+}$breast cancer, who had received lapatinib-based treatment after being diagnosed with MBC, were retrospectively analyzed at Beijing Cancer Hospital between January 2014 and November 2019. The inclusion criteria were as follows: i) HER $2^{+}$recurrent/MBC following surgery, or initial-stage IV inoperable breast cancer diagnosed by pathological biopsy. HER2 positivity was determined in the primary or metastatic focus and was defined as immunohistochemical staining of $3+$ or $2+$ with evidence of gene amplification determined by fluorescence in situ hybridization; ii) patient age of 18-80 years; iii) presence of $\geq 1$ measurable lesions, according to the Response Evaluation Criteria in Solid Tumors (RECIST) version 1.1 (14); iv) Eastern Cooperative Oncology Group performance status score of $\leq 2$; v) life expectancy of $>3$ months; and vi) no treatment contraindications. The patients received $1,250 \mathrm{mg}$ lapatinib once daily at approximately the same time every morning, with permitted dose reductions and delays for lapatinib-related toxic effects. Data on demographics, clinical outcomes and toxicity were collected based on retrospective evaluation of medical records for the descriptive analyses.

Evaluation of efficacy. All measurable lesions were recorded before lapatinib-based treatment, and imaging examination and measurements were carried out every two cycles. According to RECIST version 1.1, the evaluation of efficacy was divided into complete response (CR), partial response $(\mathrm{PR})$, stable disease (SD) and progressive disease (PD). Objective response rate $(\mathrm{ORR})=(\mathrm{CR}+\mathrm{PR}) /$ total cases; disease control rate $(\mathrm{DCR})=(\mathrm{CR}+\mathrm{PR}+\mathrm{SD}) /$ total cases; and clinical benefit rate $(\mathrm{CBR})=\mathrm{CR}+\mathrm{PR}+\mathrm{SD} \geq 6$ months/total cases Progression-free survival (PFS) was defined as the time period from the date of lapatinib-based treatment initiation until the date of first evidence of disease progression, or the date of death in the absence of disease progression. Overall survival (OS) was defined as the time period from the first day of treatment until the date of death or the date of the last follow-up. Patient follow-up generally consisted of regular physical examinations and laboratory assessments (haematological tests and serum biochemistry), left ventricular ejection fraction (LVEF) evaluation and computed tomography (CT) scans. Trastuzumab resistance was defined as recurrence/metastasis within 1 year after adjuvant therapy with trastuzumab or disease progression following the use of trastuzumab for $<6$ months in patients with MBC.

Evaluation of safety. Patients were monitored for adverse events (AEs). According to the National Cancer Institute's Common Terminology Criteria for Adverse Events (version 4.0) (15), the severity of AEs was determined as mild (grade 1), moderate (grade 2), severe (grade 3), life-threatening or disabling (grade 4), or fatal (grade 5). A cardiac event was defined as a decline in the LVEF that was symptomatic, regardless of the degree of decline, or was asymptomatic but with a relative decrease of $\geq 20 \%$ from baseline to a level below the institution's lower limit of the normal range. Lapatinib was discontinued if the patients presented with symptomatic cardiac AEs.

Statistical analysis. Data were analyzed using SPSS version 15.0 (SPSS Inc.), 'survMISC' package in $\mathrm{R}$ version 3.2.5 (http://www.r-project.org) (16) and SAS macro Renyi developed by Davis in SAS 9.4 (SAS Institute, Inc.) (17). Categorical parameters were expressed as number (percentage) of patients. Differences between groups were calculated using the $\chi^{2}$ test, where appropriate. $\mathrm{P}<0.05$ was considered to indicate a statistically significant difference. Survival was estimated by the Kaplan-Meier method. $\mathrm{P}<0.05$ (two-sided test) was considered to indicate statistical significance. Kaplan-Meier estimates of PFS and OS were completed using a log-rank test or the Renyi method (when survival curve crossover between the groups was observed). ORR and CBR were analyzed using exact methods, and a two-sided Fisher's exact test was used to compare the groups. The Cox proportional hazards model was used for multivariate analysis. $\mathrm{P}<0.05$ (two-sided test) was considered to indicate statistical significance.

\section{Results}

Patient characteristics. According to the inclusion criteria, 92 patients who had received a lapatinib-based regimen after being diagnosed with HER $2^{+} \mathrm{MBC}$ were retrospectively analyzed in the present study. The patient demographics and baseline clinical characteristics are summarized in Table I. The mean patient age was 50.5 years (range, $26-73$ years). The patients were all pathologically diagnosed with $\mathrm{HER} 2^{+}$breast cancer, with the histological types including invasive ductal carcinoma $(92.4 \%)$ and invasive lobular carcinoma $(2.2 \%)$. HER 2 positivity was detected in the primary and metastatic lesions of 86 and 6 patients, respectively. Among the 42 patients who underwent biopsy of the metastatic lesions, 31 patients $(73.8 \%)$ were HER2 ${ }^{+}$in both the primary and metastatic lesions, 5 patients (11.9\%) were HER2- in the primary but $\mathrm{HER}^{+}{ }^{+}$in the metastatic lesions, 5 patients $(11.9 \%)$ were HER2 ${ }^{+}$ in the primary but HER2- in the metastatic lesions and the HER 2 status of 1 patient (2.4\%) was unknown in the primary and HER2 $2^{+}$in the metastatic lesions. Hormone receptor positivity was defined as oestrogen and/or progesterone receptor positivity. In the present study, the hormone receptor status was positive in $48(52.2 \%)$ and negative in 44 (47.8\%) cases. The most common first metastatic sites were the lymph nodes $(43.5 \%)$, lung (41.3\%), bone (30.4\%) and liver $(29.3 \%)$, with brain as the first metastatic site in $13.0 \%$ of the patients. A total of 47 patients had 11 metastatic sites when first diagnosed with MBC, 25 patients had 2 and 20 patients had $\geq 3$.

Treatment. Among the 92 patients with HER2 ${ }^{+} \mathrm{MBC}$, $78(84.8 \%)$ were previously treated with trastuzumab in the adjuvant or recurrent/metastatic setting, 10 patients (10.9\%) received trastuzumab in the recurrent/metastatic setting after receiving a lapatinib-based regimen, and 4 patients $(4.3 \%)$ had not received trastuzumab. Different combinations of lapatinib were used: i) 68 patients $(73.9 \%)$ received lapatinib combined 
Table I. Demographic and baseline clinical characteristics of patients with human epidermal growth factor receptor-2positive metastatic breast cancer who received lapatinib-based treatment $(\mathrm{n}=92)$.

\begin{tabular}{lr}
\hline Characteristics & No. $(\%)$ \\
\hline Median age (range), years & $50.5(26-73)$ \\
Histological type & \\
Invasive ductal carcinoma & $85(92.4)$ \\
Invasive lobular carcinoma & $2(2.2)$ \\
Others & $5(5.4)$ \\
Hormone receptor status & \\
Positive & $48(52.2)$ \\
Negative & $44(47.8)$ \\
Metastatic sites & \\
Lymph nodes & $40(43.5)$ \\
Lung & $38(41.3)$ \\
Bone & $30(32.6)$ \\
Liver & $28(30.4)$ \\
Brain & $14(15.2)$ \\
Chest wall & $13(14.1)$ \\
Trastuzumab pretreatment for breast cancer & \\
Yes & $78(84.8)$ \\
Adjuvant setting & $68(73.9)$ \\
Metastatic setting & $10(10.9)$ \\
No & $14(15.2)$ \\
Current line of treatment for metastatic disease & \\
1 & $5(5.4)$ \\
2 & $11(12.0)$ \\
$\geq 3$ & \\
\hline & \\
& \\
& \\
& \\
&
\end{tabular}

with chemotherapy, including capecitabine in 38 patients, gemcitabine in 9, docetaxel in 7, paclitaxel in 5, oral etoposide in 3, vinorelbine in 3, cyclophosphamide in 2 and albumin-bound paclitaxel in 1 ; ii) 6 patients $(6.5 \%)$ received lapatinib combined with endocrine therapy, including 3 patients with letrozole, 2 patients with exemestane and 1 patient with fulvestrant; iii) 4 patients $(4.3 \%)$ received lapatinib combined with trastuzumab; iv) 10 patients $(10.9 \%)$ received lapatinib combined with chemotherapy and trastuzumab, with capecitabine used in 4 patients, paclitaxel in 2, vinorelbine in 2 , gemcitabine in 1 and docetaxel in 1 ; v) 2 patients $(2.2 \%)$ received lapatinib combined with chemotherapy followed by endocrine therapy, with docetaxel and then exemestane used in 1 case, and liposomal doxorubicin followed by fulvestrant in the other case; and vi) 2 patients $(2.2 \%)$ received lapatinib combined with endocrine therapy and trastuzumab, with letrozole used in 1 case and exemestane in the other case. Until November 30, 2019, disease progression was observed in 79 patients, and 13 patients were still receiving lapatinib treatment.

Treatment efficacy. The 92 patients were divided into three groups according to the line of lapatinib-based treatment, with
$18(19.6 \%)$ receiving first-line, $30(32.6 \%)$ second-line and $44(47.8 \%)$ third/later-line treatment. The efficacy rate, median PFS and ORR were calculated (Table II). No CR was observed among the 92 patients, whereas PR was observed in 20 (21.7\%), SD in $60(65.2 \%)$ and PD in $12(13.0 \%)$ patients, resulting in an ORR of $21.7 \%$ (20/92), DCR of $87.0 \%$ (80/92) and CBR of $47.8 \%$ (44/92). The median PFS was 5.8 months [95\% confidence interval (CI): 4.9-6.8] and the median OS 21.5 months (95\% CI: 19.7-23.3).

In the three groups of patients receiving lapatinib-based regimen as first-, second- and third/later-line treatment, the median PFS was 10.4, 5.2 and 5.1 months, the median OS was 32.9, 29.1 and 13.0 months, the ORR was 38.9, 23.3 and $13.60 \%$, and the DCR was $100,83.3$ and $84.1 \%$, respectively. Differences in the median PFS among the three groups were calculated and found to be statistically significant $(\mathrm{P}=0.048)$. Differences in the median OS among the three groups were also found to be statistically significant. The $\chi^{2}$ test was used to compare the distribution of therapeutic efficacy in the three groups, with the results exhibiting no statistically significant difference. A survival curve was drawn using the Kaplan-Meier PFS estimates (Fig. 1). Among the 92 patients with HER2 ${ }^{+}$MBC, 71 were trastuzumab-resistant. In the trastuzumab-resistant $(n=71)$ and trastuzumab-sensitive $(n=21)$ patients, the median PFS was 5.2 and 9.1 months, and the median OS was 21.4 and 44.3 months, respectively. Differences in the median PFS $(\mathrm{P}=0.032)$ and $\mathrm{OS}(\mathrm{P}=0.003)$ between the two groups were statistically significant. A survival curve was drawn using the Kaplan-Meier PFS and OS estimates (Fig. 2).

Different treatment combinations. The 92 patients were divided into several groups according to the different agents combined with lapatinib: i) The median PFS of the groups of patients receiving lapatinib combined with chemotherapy $(n=70)$, endocrine therapy $(\mathrm{n}=6)$ and targeted therapy $(\mathrm{n}=16)$ was 5.9 (95\% CI: 4.1-7.7), 5.1 (95\% CI: 4.1-6.1) and 5.9 (95\% CI: 1.5-10.2) months, respectively. The P-value was 0.878 when comparing the median PFS among these groups. ii) The median PFS of the two groups of patients receiving lapatinib combined with targeted $(n=16)$ and non-targeted $(n=76)$ therapy was 5.9 (95\% CI: 1.5-10.2) and 5.7 (95\% CI: 4.7-6.7) months, respectively $(\mathrm{P}=0.903)$. iii) The median $\mathrm{PFS}$ of the two groups of patients receiving lapatinib combined with chemotherapy $(n=76)$ and chemotherapy plus trastuzumab $(n=10)$ was 5.9 (95\% CI: 4.5-7.3) and 5.9 (95\% CI: 2.2-9.5) months, respectively $(\mathrm{P}=0.239)$. Among the 92 patients, 68 received lapatinib in combination with chemotherapy alone. The median PFS of these 68 patients was 5.9 (95\% CI: 4.5-7.3) months, while the median PFS of the three groups was 10.4 (95\% CI: 7.9-12.8) months in the first-line $(n=17), 4.6$ months $(95 \%$ CI: 3.5-5.6) months in the second-line $(n=20)$ and 5.0 (95\% CI: 3.5-6.6) months in the third/later-line $(n=31)$ setting. The median PFS differed significantly among these three groups $(\mathrm{P}=0.027)$.

Capecitabine vs. other chemotherapy agents. Among the 68 patients who received lapatinib plus chemotherapy alone, 38 received capecitabine (12 cases in the first-line, 13 cases in the second-line and 13 cases in the third/later-line setting), and 30 cases received other chemotherapy agents. The patients 
Table II. Efficacy of lapatinib-based treatment in patients with human epidermal growth factor receptor-2-positive breast cancer $(n=92)$.

\begin{tabular}{|c|c|c|c|c|}
\hline Efficacy measures & First-line & Second-line & Third/later-line & Total \\
\hline Median PFS (95\% CI), months & $10.4(7.9-12.8)$ & $5.2(2.8-7.6)$ & $5.1(4.3-5.9)$ & $5.8(4.9-6.8)$ \\
\hline Median OS $(95 \% \mathrm{CI})$, months & $32.9(6.3-59.4)$ & $29.1(18.0-40.3)$ & $13.0(1.3-24.7)$ & $21.5(19.7-23.3)$ \\
\hline Objective response rate, $\%$ & 38.9 & 23.3 & 13.6 & 21.7 \\
\hline Disease control rate, $\%$ & 100 & 83.3 & 84.1 & 87.0 \\
\hline Partial response, $\mathrm{n}$ & 7 & 7 & 6 & 20 \\
\hline Stable disease, $\mathrm{n}$ & 11 & 18 & 31 & 60 \\
\hline Progressive disease, $\mathrm{n}$ & 0 & 5 & 7 & 12 \\
\hline
\end{tabular}

PFS, progression-free survival; OS, overall survival; CI, confidence interval.
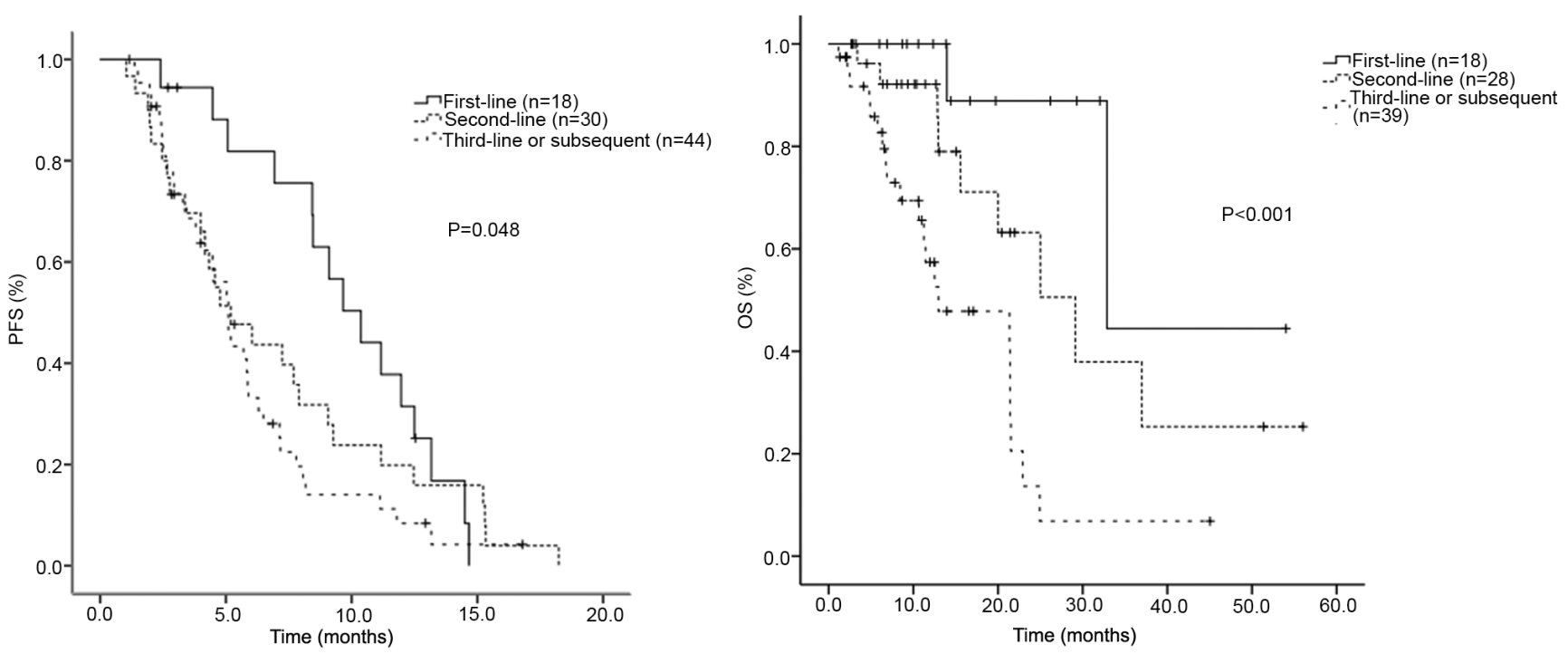

Figure 1. PFS and OS with different lines of lapatinib-based treatment. PFS, progression-free survival; OS, overall survival.

receiving lapatinib plus capecitabine $(n=38)$ had a prolonged PFS of 8.1 (95\% CI: 4.3-11.8) months, compared with patients receiving lapatinib plus other chemotherapy agents $(n=30)$ with a median PFS of 5.1 months (95\% CI: 2.6-7.5 months; $\mathrm{P}=0.005$; Fig. 3A). In the first-line setting, the median PFS of the patients receiving lapatinib combined with capecitabine $(n=12)$ and other chemotherapy agents $(n=5)$ was 12.0 (95\% CI: 8.3-15.6) and 8.4 (95\% CI: 1.2-15.7) months, respectively. The patients receiving lapatinib combined with capecitabine exhibited longer PFS compared with patients receiving lapatinib combined with other chemotherapy agents $(\mathrm{P}=0.019$; Fig. 3B). In the second-line setting, the median PFS of the patients receiving lapatinib combined with capecitabine $(n=13)$ and other chemotherapy agents $(n=7)$ was 4.2 (95\% CI: 2.9-5.5) and 5.2 (95\% CI: 3.6-6.8) months, respectively. The median PFS did not differ significantly between these two groups $(\mathrm{P}=0.901$; Fig. 3C). In the third/later-line setting, the median PFS of the patients receiving lapatinib combined with capecitabine $(n=13)$ and other chemotherapy agents $(n=18)$ was $6.3(95 \%$ CI: 4.2-8.4) and 3.3 (95\% CI: 2.0-4.6) months, respectively. The patients receiving lapatinib combined with capecitabine had a longer PFS compared with the patients receiving lapatinib combined with other chemotherapy agents $(\mathrm{P}=0.015$; Fig. 3D). Survival curves were drawn by Kaplan-Meier PFS estimates in these different groups (Fig. 3).

Of the 38 patients who received lapatinib plus capecitabine, 12 had received capecitabine prior to lapatinib, and the remaining 26 patients had received capecitabine first. The median PFS of these two groups of patients was 6.3 (95\% CI: 2.1-10.5) and 9.1 (95\% CI: 4.3-13.8) months, respectively $(\mathrm{P}=0.945)$.

Brain metastasis. In the present study, 14 patients with brain metastasis from HER2 ${ }^{+} \mathrm{MBC}$ were treated with lapatinib at the recurrence and metastasis stage. Among the 14 patients with brain metastasis who had received lapatinib-based treatment, 6 had received lapatinib as first-line, 5 as second-line, 2 as third-line and 1 as fourth-line treatment. Lapatinib was combined with capecitabine $(n=5)$, docetaxel $(n=3)$, capecitabine plus trastuzumab $(n=2)$, trastuzumab $(n=1)$, paclitaxel $(n=1)$, capecitabine plus gemcitabine $(n=1)$, and 

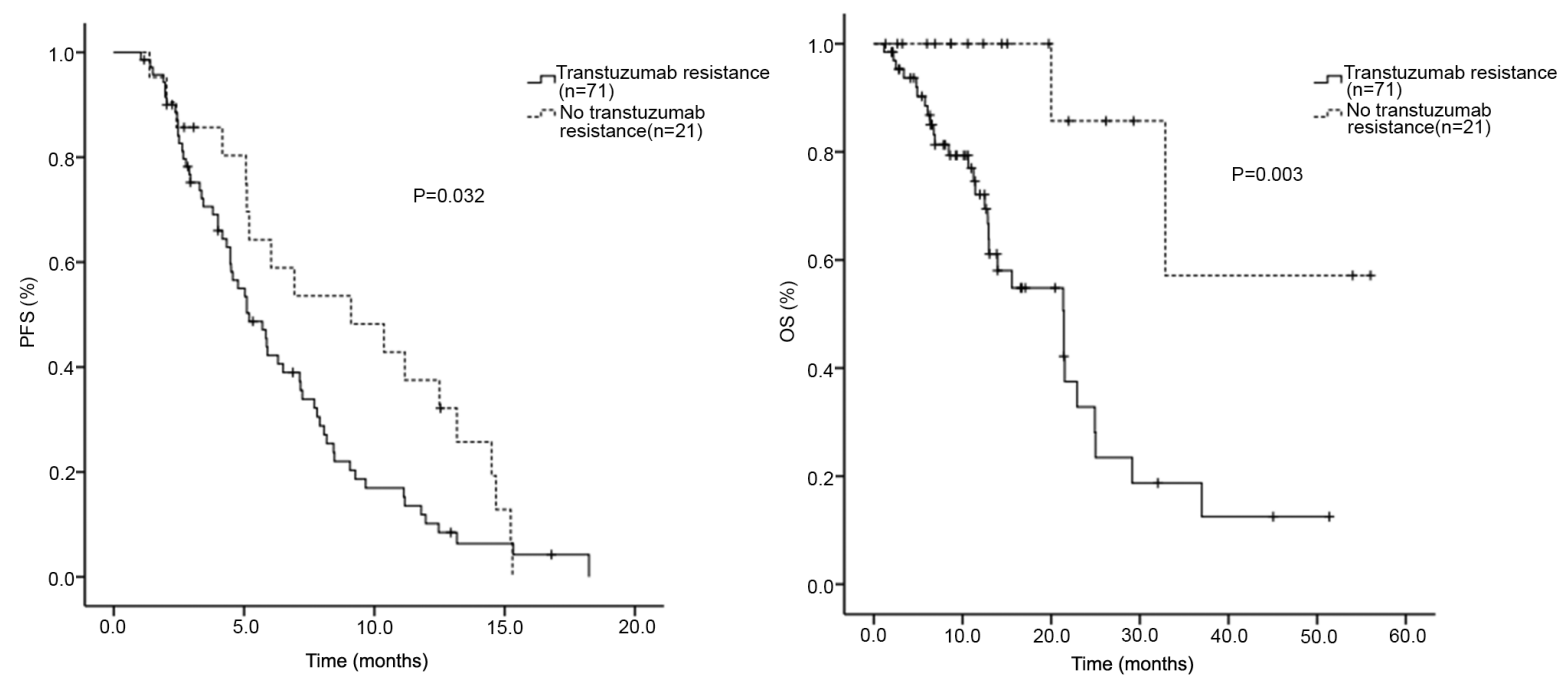

Figure 2. PFS and OS in trastuzumab-resistant $(\mathrm{n}=71)$ and trastuzumab-sensitive $(\mathrm{n}=21)$ patients. PFS, progression-free survival; OS, overall survival.
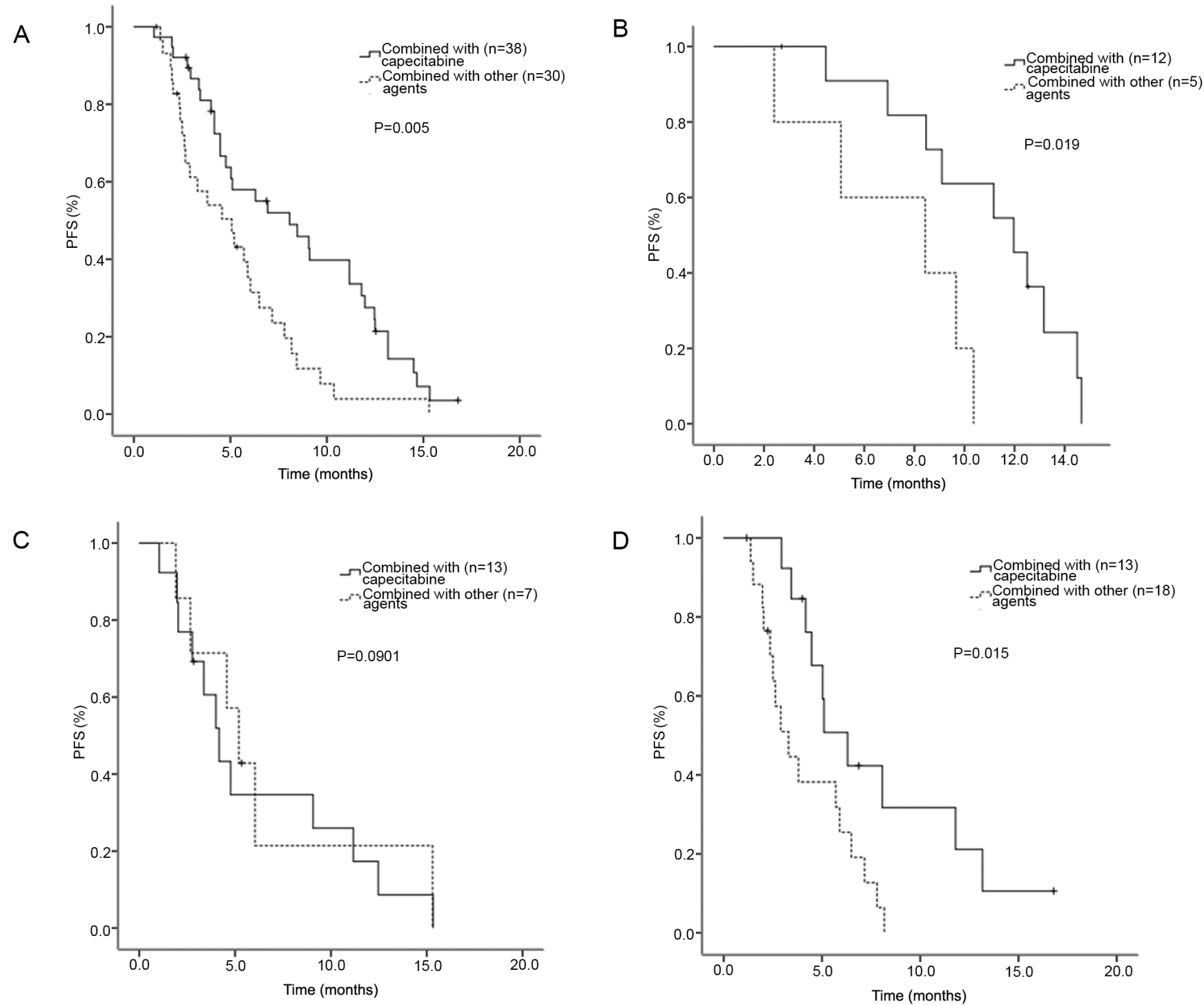

Figure 3. PFS in patients receiving lapatinib plus capecitabine, and those receiving lapatinib plus other chemotherapy agents in different lines of treatment. Comparison between (A) patients receiving lapatinib combined with capecitabine $(\mathrm{n}=38)$ and those receiving other chemotherapy agents $(\mathrm{n}=30)$, (B) patients receiving lapatinib combined with capecitabine $(n=12)$ and those receiving other chemotherapy agents $(n=5)$ as first-line treatment, (C) patients receiving lapatinib combined with capecitabine $(n=13)$ and those receiving other chemotherapy agents $(n=7)$ as second-line treatment, and (D) patients receiving lapatinib combined with capecitabine $(n=13)$ and those receiving other chemotherapy agents $(n=18)$ as third/later-line treatment. PFS, progression-free survival. 
Table III. Multivariate analysis of the independent prognostic factors of median progression-free survival of patients with human epidermal growth factor receptor-2-positive metastatic breast cancer receiving lapatinib-based treatment (n=92).

\begin{tabular}{lccc}
\hline Prognostic factors & P-value & HR & 95\% CI \\
\hline Line of lapatinib-based treatment & $0.024^{\mathrm{a}}$ & 1.55 & $1.06-2.268$ \\
Combination with capecitabine & $0.009^{\mathrm{a}}$ & 2.288 & $1.234-4.245$ \\
Presence of liver metastases & 0.29 & 1.427 & $0.739-2.758$ \\
Presence of brain metastases & 0.392 & 1.437 & $0.626-3.3$ \\
Presence of visceral metastases & 0.462 & 0.764 & $0.373-1.564$ \\
Number of metastatic sites & 0.341 & 0.82 & $0.545-1.234$ \\
Hormone receptor status & 0.251 & 1.396 & $0.79-2.468$ \\
\hline
\end{tabular}

${ }^{\mathrm{a}} \mathrm{P}<0.05$. HR, hazard ratio; $\mathrm{CI}$, confidence interval.

Table IV. Lapatinib-related adverse effects and dose reduction.

\begin{tabular}{lccccc}
\hline Adverse effects & Grade 1 & Grade 2 & Grade 3 & Grade 4 & Grade 5 \\
\hline Diarrhoea & 4 & $11^{\mathrm{a}}$ & $1^{\mathrm{a}}$ & 0 & 0 \\
Rash & 6 & 3 & 0 & 0 & 0 \\
Hand-foot syndrome & 2 & $1^{\mathrm{a}}$ & $1^{\mathrm{a}}$ & 0 & 0 \\
Dose reduction & & $12^{\mathrm{a}}$ & $2^{\mathrm{a}}$ & & \\
\hline
\end{tabular}

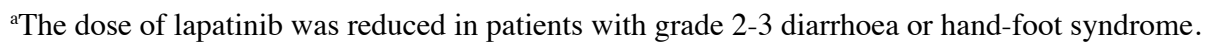

vinorelbine plus trastuzumab $(n=1)$. All patients with brain metastasis received radiotherapy. The follow-up results revealed a median PFS of 8.4 (95\% CI: 2.2-14.7) months in 14 patients with brain metastases. The number of patients with PR, SD and PD was 5, 7 and 2, respectively, with an ORR of $35.7 \%$ and a DCR of $85.7 \%$. When comparing the median PFS between patients with $(n=14)$ and without $(n=78)$ brain metastasis, the difference was not statistically significant $(\mathrm{P}=0.569)$.

Multivariate analysis. Multivariate logistic regression analysis was performed to evaluate the independent prognostic factors of median PFS in patients with HER2 ${ }^{+}$MBC receiving lapatinib-based treatment, and revealed that the line of lapatinib-based treatment [hazard ratio $(\mathrm{HR})=1.55$; 95\% CI: $1.1-2.3 ; \mathrm{P}=0.024)$ and its combination with capecitabine $(\mathrm{HR}=2.288 ; 95 \% \mathrm{CI}: 1.2-4.2 ; \mathrm{P}=0.009)$ were strong predictors of the median PFS of patients with HER2 ${ }^{+}$MBC (Table III).

AEs. The lapatinib-based combination treatment was generally well-tolerated. The most common lapatinib-related AEs were diarrhoea, rash and hand-foot syndrome. Diarrhoea was recorded in 16 patients (4 cases of grade 1, 11 cases of grade 2 and 1 case of grade 3 ). Rash was recorded in 9 patients (6 cases of grade 1 and 3 cases of grade 2). Hand-foot syndrome was recorded in 4 patients ( 2 cases of grade 1,1 case of grade 2 and 1 case of grade 3 ). There was no episode of febrile neutropenia or symptomatic cardiac events (Table IV). The dose of lapatinib was reduced in
14 patients, of whom 12 had diarrhoea and 2 had hand-foot syndrome (grades 2-3).

\section{Discussion}

Anti-HER 2 agents are supposed to be used soon after the diagnosis of HER $2^{+}$MBC. Novel and emerging agents targeting HER2 and its pathway are associated with notable clinical benefits in patients having received treatment with trastuzumab. Lapatinib was approved for use in combination with capecitabine in patients with HER2 ${ }^{+} \mathrm{MBC}$ who had received prior treatment, including treatment with an anthracycline, a taxane and trastuzumab, in 2007 (8). Subsequent research explored the efficacy and tolerability of lapatinib in different treatment phases of HER2 ${ }^{+} \mathrm{MBC}$, and demonstrated that lapatinib-based combination regimens may be beneficial for heavily pretreated patients with HER2 ${ }^{+}$MBC (18).

The present study is a real-world study that aimed to determine the efficacy and tolerability of the lapatinib-based treatment in a Chinese HER2 ${ }^{+}$MBC patient population. According to the findings, lapatinib-based combination treatment was effective and well-tolerated, with a median PFS of 5.8 months, a median OS of 21.5 months, an ORR of $21.7 \%$, a DCR of $87.0 \%$ and limited AEs. A lapatinib-based regimen was used as first-line treatment in 18 patients who progressed following treatment with trastuzumab as adjuvant therapy. The median PFS was 10.4 months, the median OS was 32.9 months, the ORR was $38.9 \%$ and the DCR was $100 \%$, which clearly demonstrated the benefits of lapatinib, and supported its use combined with chemotherapy as a first-line alternative for 
patients with trastuzumab-resistant HER $2^{+}$MBC. Similarly, the EMILIA trial (10) randomized patients with $\mathrm{HER}^{+} \mathrm{MBC}$ who had been pretreated with trastuzumab and taxane to receive trastuzumab emtansine (T-DM1) or lapatinib plus capecitabine. The results revealed a median PFS of 9.6 months with T-DM1, compared with 6.4 months with lapatinib plus capecitabine (HR=0.65; 95\% CI: 0.55-0.77; $\mathrm{P}<0.001)$. Trastuzumab plus chemotherapy or endocrine therapy is commonly used at present as first-line treatment for HER2 ${ }^{+} \mathrm{MBC}$. However, lapatinib combined with chemotherapy may be another option for trastuzumab-pretreated patients, since T-DM1 was not approved until January, 2020 in China.

In the present study, a lapatinib-based regimen was used in 30 patients as second-line treatment (median PFS, 5.2 months; median OS, 29.1 months; ORR, 23.3\%; DCR, 83.3\%) and in 44 patients as third/later-line treatment (median PFS, 5.1 months; median OS, 13.0 months; ORR, 13.6\%; DCR, 84.1\%), which confirmed the survival benefits of lapatinib when used as second/later-line treatment. Previous studies reported similar results following treatment with lapatinib plus capecitabine or trastuzumab. Bian et al (19) compared lapatinib plus capecitabine with trastuzumab plus capecitabine in trastuzumab-resistant and taxane-pretreated patients with HER $2^{+} \mathrm{MBC}$, and revealed a significantly improved PFS (6.0 vs. 4.5 months) and higher proportion of patients with a PFS of $\geq 6$ months (55 vs. $30 \%$; $\mathrm{P}=0.005)$. In the EGF100151 study $(20,21)$, lapatinib plus capecitabine significantly prolonged median PFS, and reduced the risk of disease progression and death, when compared with capecitabine alone, with a controllable toxicity and good tolerance. Furthermore, the risk of disease progression was lower when using lapatinib plus capecitabin as second-line treatment, as compared to third/later-line treatment. The EGF104900 study (22) demonstrated that trastuzumab combined with lapatinib may achieve a better PFS (prolonged by 3.9 months) and OS compared with lapatinib alone in patients with HER2 ${ }^{+}$MBC. Baez-Vallecillo et al (23) analyzed 520 patients with HER2 ${ }^{+} \mathrm{MBC}$ who had received prior treatment with trastuzumab, pertuzumab and/or ado-T-DM1 and then lapatinib, and revealed a median treatment duration of lapatinib of 5.0 months, a median time-to-progression (TTP) of 6.0 months, and a CBR of $28 \%$. The present study demonstrated that the median PFS of the trastuzumab-resistant $(n=71)$ and trastuzumab-sensitive $(n=21)$ patients was 5.2 and 9.1 months, and the median OS was 21.4 and 44.3 months, respectively. Based on the present findings and the aforementioned research, a lapatinib-based combination (including lapatinib combined with chemotherapy or trastuzumab) appears to be a superior alternative, particularly in trastuzumab-pretreated patients with HER2 ${ }^{+}$MBC. The results of the present study also suggested that the earlier use of the lapatinib combination was correlated with a better PFS (24).

The patients were divided into different groups according to the different combination treatments with lapatinib, and no significant difference in the median PFS was observed among the patients receiving lapatinib combined with chemotherapy, endocrine therapy and targeted therapy, lapatinib combined with targeted and non-targeted therapy, and lapatinib combined with chemotherapy and chemotherapy plus trastuzumab. Thus, various combinations may be taken into consideration when using lapatinib. Based on our experience, lapatinib plus endocrine therapy or trastuzumab may also be considered for older patients ( $>65$ years old) who are in a relatively poor condition and unable to tolerate chemotherapy.

In the present study, of the 68 patients who received lapatinib plus chemotherapy alone, 38 received capecitabine and 30 other chemotherapy agents. A total of 23/30 (76.7\%) patients received lapatinib combined with other chemotherapy drugs, since they had developed PD on capecitabine before, and $3 / 30(10 \%)$ patients received lapatinib combined with other chemotherapy drugs, which had shown good efficacy as previous treatments. A significant difference was observed in the whole group, and the first-line and third/later-line subgroups, with patients receiving lapatinib combined with capecitabine exhibiting a significantly improved PFS compared with those receiving lapatinib combined with other chemotherapy agents. The results were consistent with the evidence on lapatinib combined with capecitabine in HER2 ${ }^{+}$MBC reported by several clinical trials. Capecitabine in combination with lapatinib is an acceptable treatment option for patients, since the agents may be taken orally at home, are associated with limited AEs and a small effect on immune function, contributing to an optimal quality of life for these patients. In addition, the pharmacological mechanisms underlying the positive interaction between lapatinib and capecitabine were investigated in human breast cancer models, and it was observed that lapatinib clearly downregulated thymidylate synthase (TS) activity, thereby improving the efficacy of capecitabine, and that capecitabine optimized the downregulation of $\mathrm{p}$-AKT and p-P42/44 expression by lapatinib. Specifically, lapatinib and capecitabine modulated each other's molecular determinants of response, and concomitant dosing appeared to be the optimal method for combining these agents, which suggested that the association between lapatinib and capecitabine has the potential to overcome breast cancer resistance associated with TS overexpression (25). Therefore, lapatinib plus capecitabine may be considered as a superior combination for patients with HER $2^{+}$MBC due to its efficacy, convenience and tolerability. It was hypothesized that the reason for the lack of significant differences in the second-line subgroup was due to the limited number of cases. In addition, the prior use of capecitabine did not affect the median PFS.

Among patients with MBC, 6-16\% will develop metastatic CNS disease $(2,5)$. However, brain metastasis may be underdiagnosed without routine screening, due to the lack of clinical symptoms. Lapatinib is able to cross the blood-brain barrier and concentrate in HER $2^{+} \mathrm{MBC}$, but not in normal brain tissue, due to HER2 selectivity (26-28). Patients with brain metastasis must receive appropriate radiotherapy and systemic treatment. In the present study, the median PFS of the 14 patients with brain metastases from HER2 ${ }^{+}$MBC was 8.4 months, the ORR was $35.7 \%$ and the DCR was $85.7 \%$, which suggested that lapatinib may be a viable therapeutic option for such patients. In the phase II LANDSCAPE study (9), lapatinib plus capecitabine was effective in patients with previously untreated brain metastases from HER2 ${ }^{+} \mathrm{MBC}$, with an objective CNS response of $65.9 \%$ (95\% CI: 50.1-79.5) and a median TTP of 5.5 months (95\% CI: 4.3-6.0), which supported the use of lapatinib plus capecitabine as first-line treatment for patients with brain metastases from HER $2^{+}$breast cancer. 
A study from Turkey (29) also demonstrated that lapatinib plus capecitabine treatment conferred a significant survival benefit to patients with brain metastasis from breast cancer, as compared with trastuzumab-based treatment. The present analysis confirmed the benefits of lapatinib in patients with brain metastases from HER $2^{+}$breast cancer.

Multivariate logistic regression analysis revealed that the line of lapatinib-based treatment and its combination with capecitabine were independent prognostic factors for the median PFS of patients with HER2 ${ }^{+} \mathrm{MBC}$, which supports the earlier use of lapatinib and its combination with capecitabine.

There were certain limitations to the present study. The included patients represented a highly selected population referred to tertiary care cancer centres, limiting the ability to generalize from the present results. Large-scale prospective clinical trials are required to elucidate the therapeutic effect and safety of lapatinib plus capecitabine in HER2 ${ }^{+} \mathrm{MBC}$.

In conclusion, lapatinib-based treatment was found to be effective and well-tolerated by patients with HER $2^{+} \mathrm{MBC}$ (even trastuzumab-pretreated patients), particularly when combined with capecitabine.

\section{Acknowledgements}

Not applicable.

\section{Funding}

No funding was received.

\section{Availability of data and materials}

All the datasets used and/or analyzed during the present study are available from the corresponding author on reasonable request.

\section{Authors' contributions}

XG, HL, YY and RL contributed to the study conception and design. Material preparation and data collection were performed by XG and YY, and XG, RZ and HL analyzed and interpreted the patient data. The draft of the manuscript was written by XG and revised by PL. XG, HL, YY and RL commented on previous versions of the manuscript. All the authors have read and approved the final manuscript. Each of the authors has sufficiently participated in the work to take public responsibility for appropriate parts of the content and agrees to be accountable for all aspects of the work to ensure that questions regarding the accuracy or integrity of any part of the work are appropriately investigated and resolved.

\section{Ethics approval and consent to participate}

The study protocol was reviewed and approved by the Ethics Committee of Peking University Cancer Hospital and Institute.

\section{Patient consent for publication}

Not applicable.

\section{Competing interests}

All authors declare that they have no competing interests.

\section{References}

1. Lim B, Murthy RK, Lee J, Jackson SA, Iwase T, Davis DW, Willey JS, Wu J, Shen Y, Tripathy D, et al: A phase Ib study of entinostat plus lapatinib with or without trastuzumab in patients with HER2-positive metastatic breast cancer that progressed during trastuzumab treatment. Br J Cancer 120: 1105-1112, 2019.

2. Leyland-Jones B: Human epidermal growth factor receptor 2-positive breast cancer and central nervous system metastases. J Clin Oncol 27: 5278-5286, 2009.

3. Kaal EC and Vecht CJ: CNS complications of breast cancer: Current and emerging treatment options. CNS Drugs 21: 559-579, 2007.

4. Gabos Z, Sinha R, Hanson J, Chauhan N, Hugh J, Mackey JR and Abdulkarim B: Prognostic significance of human epidermal growth factor receptor positivity for the development of brain metastasis after newly diagnosed breast cancer. J Clin Oncol 24: 5658-5663, 2006.

5. Pieńkowski $\mathrm{T}$ and Zielinski CC: Trastuzumab treatment in patients with breast cancer and metastatic CNS disease. Ann Oncol 21: 917-924, 2010.

6. Moy B and Goss PE: Lapatinib-associated toxicity and practical management recommendations. Oncologist 12: 756-765, 2007.

7. Murphy CG and Modi S: HER2 breast cancer therapies: A review. Biologics 3: 289-301, 2009.

8. Ryan Q, Ibrahim A, Cohen MH, Johnson J, Ko CW, Sridhara R, Justice R and Pazdur R: FDA drug approval summary: Lapatinib in combination with capecitabine for previously treated metastatic breast cancer that overexpresses HER-2. Oncologist 13: 1114-1119, 2008.

9. Bachelot T, Romieu G, Campone M, Diéras V, Cropet C Dalenc F, Jimenez M, Le Rhun E, Pierga JY, Gonçalves A, et al: Lapatinib plus capecitabine in patients with previously untreated brain metastases from HER2-positive metastatic breast cancer (LANDSCAPE): A single-group phase 2 study. Lancet Oncol 14: 64-71, 2013.

10. Verma S, Miles D, Gianni L, Krop IE, Welslau M, Baselga J, Pegram M, Oh DY, Diéras V, Guardino E, et al: Trastuzumab emtansine for HER2-positive advanced breast cancer. N Engl J Med 367: 1783-1791, 2012.

11. Johnston S, Pippen J Jr, Pivot X, Lichinitser M, Sadeghi S, Dieras V, Gomez HL, Romieu G, Manikhas A, Kennedy MJ, et al: Lapatinib combined with letrozole versus letrozole and placebo as first-line therapy for postmenopausal hormone receptor-positive metastatic breast cancer. J Clin Oncol 27: 5538-5546, 2009.

12. Lin NU, Diéras V, Paul D, Lossignol D, Christodoulou C, Stemmler HJ, Roché H, Liu MC, Greil R, Ciruelos E, et al: Multicenter phase II study of lapatinib in patients with brain metastases from HER2-positive breast cancer. Clin Cancer Res 15: 1452-1459, 2009.

13. Bachelot T, Le Rhun E, Labidi-Gally I, Heudel P, Gilabert M, Bonneterre J, Pierga JY and Gonçalves A: Systemic treatment of brain metastases from breast cancer: Cytotoxic chemotherapy and targeted therapies. Bull Cancer 100: 7-14, 2013.

14. Eisenhauer EA, Therasse P, Bogaerts J, Schwartz LH, Sargent D, Ford R, Dancey J, Arbuck S, Gwyther S, Mooney M, et al: New response evaluation criteria in solid tumours: Revised RECIST guideline (version 1.1). Eur J Cancer 45: 228-247, 2009.

15. NCI: CTCAE. URL:http://evs.nci.nih.gov.cmich.idm.oclc. org/ftp1/CTCAE/about.html. Accessed May 17, 2010.

16. Royston P: Explained variation for survival models. Stata J 6: 83-96, 2006.

17. Davis $M$ and Xie SX: Caution: Hazards crossing! Using the Renyi test statistic in survival analysis. PharmaSUG2011-Paper SP06, 2011. https://www.pharmasug.org/proceedings/2011/SP/ PharmaSUG-2011-SP06.pdf.

18. Greil R, Borštnar S, Petráková K, Marcou Y, Pikiel J, Wojtukiewicz MZ, Koza I, Steger GG, Linn M, Das Gupta A and Cwiertka K: Combination therapy of lapatinib and capecitabine for ErbB2-positive metastatic or locally advanced breast cancer: Results from the lapatinib expanded access program (LEAP) in central and Eastern Europe. Onkologie 34: 233-238, 2011. 
19. Bian L, Wang T, Zhang S and Jiang Z: Trastuzumab plus capecitabine vs lapatinib plus capecitabine in patients with trastuzumab resistance and taxane-pretreated metastatic breast cancer. Tumour Biol 34: 3153-3158, 2013.

20. Cameron D, Casey M, Press M, Lindquist D, Pienkowski T, Romieu CG, Chan S, Jagiello-Gruszfeld A, Kaufman B, Crown J, et al: A phase III randomized comparison of lapatinib plus capecitabine versus capecitabine alone in women with advanced breast cancer that has progressed on trastuzumab: Updated efficacy and biomarker analyses. Breast Cancer Res Treat 112: 533-543, 2008

21. Geyer CE, Forster J, Lindquist D, Chan S, Romieu CG, Pienkowski T, Jagiello-Gruszfeld A, Crown J, Chan A, Kaufman B, et al: Lapatinib plus capecitabine for HER2-positive advanced breast cancer. N Engl J Med 355: 2733-2743, 2006

22. Blackwell KL, Burstein HJ, Storniolo AM, Rugo HS, Sledge G, Aktan G, Ellis C, Florance A, Vukelja S, Bischoff J, et al: Overall survival benefit with lapatinib in combination with trastuzumab for patients with human epidermal growth factor receptor 2-positive metastatic breast cancer: Final results from the EGF104900 study. J Clin Oncol 30: 2585-2592, 2012.

23. Baez-Vallecillo L, Raghavendra AS, Hess KR, Barcenas $\mathrm{CH}$, Moulder SL, Tripathy D, Valero V and Murthy RK: Lapatinib activity in metastatic human epidermal growth factor receptor 2-positive breast cancers that received prior therapy with trastuzumab, pertuzumab, and/or ado-trastuzumab emtansine (T-DM1). Breast Cancer Res Treat 176: 227-234, 2019.

24. Untch M and Lück HJ: Lapatinib-member of a new generation of ErbB-targeting drugs. Breast Care (Basel) 5: 8-12, 2010.

25. Chefrour M, Milano G, Formento P, Giacometti S, Denden A, Renée N, Iliadis A, Fischel JL and Ciccolini J: Positive interaction between lapatinib and capecitabine in human breast cancer models: Study of molecular determinants. Fundam Clin Pharmacol 26: 530-537, 2012.
26. Gril B, Palmieri D, Bronder JL, Herring JM, Vega-Valle E, Feigenbaum L, Liewehr DJ, Steinberg SM, Merino MJ, Rubin SD and Steeg PS: Effect of lapatinib on the outgrowth of metastatic breast cancer cells to the brain. J Natl Cancer Inst 100: 1092-1103, 2008.

27. Saleem A, Searle GE, Kenny LM, Huiban M, Kozlowski K, Waldman AD, Woodley L, Palmieri C, Lowdell C, Kaneko T, et al: Lapatinib access into normal brain and brain metastases in patients with Her-2 overexpressing breast cancer. EJNMMI Res 5: 30, 2015.

28. Morikawa A, Peereboom DM, Thorsheim HR, Samala R, Balyan R, Murphy CG, Lockman PR, Simmons A, Weil RJ, Tabar V, et al: Capecitabine and lapatinib uptake in surgically resected brain metastases from metastatic breast cancer patients: A prospective study. Neuro Oncol 17: 289-295, 2015.

29. Kaplan MA, Isikdogan A, Koca D, Kucukoner M, Gumusay O, Yildiz R, Dayan A, Demir L, Geredeli C, Kocer M, et al: Clinical outcomes in patients who received lapatinib plus capecitabine combination therapy for HER2-positive breast cancer with brain metastasis and a comparison of survival with those who received trastuzumab-based therapy: A study by the Anatolian society of medical oncology. Breast Cancer 21: 677-683, 2014.

This work is licensed under a Creative Commons Attribution-NonCommercial-NoDerivatives 4.0 International (CC BY-NC-ND 4.0) License. 